\title{
Structure and Dynamics of Dinucleosomes Assessed by Atomic Force Microscopy
}

\author{
Nina A. Filenko, ${ }^{1,2}$ Dmytro B. Palets, ${ }^{1,3}$ and Yuri L. Lyubchenko ${ }^{1}$ \\ ${ }^{1}$ Department of Pharmaceutical Sciences, University of Nebraska Medical Center, Omaha, NE 68198-6025, USA \\ ${ }^{2}$ Department of High Technologies, The International Solomon University, 1b Sholudenko Street, Kyiv 01135, Ukraine \\ ${ }^{3}$ Department of Application Services, Infopulse Ukraine LLC, 24 Polyova Street, Kyiv 03056, Ukraine
}

Correspondence should be addressed to Yuri L. Lyubchenko, ylyubchenko@unmc.edu

Received 21 June 2011; Accepted 13 August 2011

Academic Editor: Kunchithapadam Swaminathan

Copyright (C) 2012 Nina A. Filenko et al. This is an open access article distributed under the Creative Commons Attribution License, which permits unrestricted use, distribution, and reproduction in any medium, provided the original work is properly cited.

Dynamics of nucleosomes and their interactions are important for understanding the mechanism of chromatin assembly. Internucleosomal interaction is required for the formation of higher-order chromatin structures. Although $\mathrm{H} 1 \mathrm{histone}$ is critically involved in the process of chromatin assembly, direct internucleosomal interactions contribute to this process as well. To characterize the interactions of nucleosomes within the nucleosome array, we designed a dinucleosome and performed direct AFM imaging. The analysis of the AFM data showed dinucleosomes are very dynamic systems, enabling the nucleosomes to move in a broad range along the DNA template. Di-nucleosomes in close proximity were observed, but their population was low. The use of the zwitterionic detergent, CHAPS, increased the dynamic range of the di-nucleosome, facilitating the formation of tight di-nucleosomes. The role of CHAPS and similar natural products in chromatin structure and dynamics is also discussed.

\section{Introduction}

The formation of nucleosomes is the first stage of DNA packing into chromatin, followed by the assembly of the "beadson-a-string" nucleosomal array into compact chromatin fibers (e.g., [1] and references therein). H1 histone is a key player in the formation of the 30-nm-thick fibers (e.g., [2]); however, interactions between the nucleosomal particles contribute to the assembly process as well.

Although the molecular mechanisms behind the formation of higher-order chromatin structures remain unclear, work employing model systems has shown that in vitro reconstituted nucleosome arrays containing only DNA and core histone proteins undergo the same initial salt-dependent condensations as native chromatin $[1,3]$. In solutions containing physiological concentrations of monoand divalent cations, nucleosome arrays spontaneously fold into structures with the same hydrodynamic shape as the 30 -nm-diameter chromatin fiber $[1,4]$. This implies that the primary protein determinants defining these structures reside within the core histone proteins. Indeed, in the early crystallography work of Luger et al. [5], it was shown that the K16 to N25 segment of $\mathrm{H} 4$ makes extensive contacts with an H2A-H2B dimer of an adjacent particle. This $\mathrm{H} 4$ region, K16 to N25, makes multiple hydrogen bonds and salt bridges between its basic side chains (K16, R19, K20, R23) and acidic side chains of H2A (E56, E61, E64, D90, E91, E92) and $\mathrm{H} 2 \mathrm{~B}$ (E110). These structure-based predictions were supported with studies [6-8] of recombinant core mutants. They showed that interaction between the $\mathrm{H} 4 \mathrm{~N}$-terminal domain (NTD) and a surface-exposed $\mathrm{H} 2 \mathrm{~A}$ region on a neighboring nucleosome is required for assembly of folded 30-nm secondary structures. More importantly, nucleosomal arrays lacking all core histone NTDs are unable to fold into the 30-nm secondary chromatin structures [3, 9], even in the presence of bound linker histones [10]. This data shows that interaction between nucleosomes in the absence of $\mathrm{H} 1$ takes place. Results indicate that all core histone Nterminal domains (NTD) participate in the oligomerization process and that the NTDs function additively and independently [11]. According to the Monte Carlo simulations [12], the $\mathrm{H} 4$ tails mediate the majority of the internucleosomal 
interactions, followed by the $\mathrm{H} 3, \mathrm{H} 2 \mathrm{~A}$, and $\mathrm{H} 2 \mathrm{~B}$ tails in decreasing order. The $\mathrm{H} 4$ histone role was also studied separately and was found that the N-terminal end of the H4 tail participates in intra- and internucleosomal interactions with protein and DNA during folding and oligomerization of nucleosome arrays [13].

Atomic force microscopy (AFM) was applied in [14] to study interactions between nucleosomes within the assembled "beads-on-a-string" nucleosomal array in the absence of $\mathrm{H} 1$ histone. The experimental data were supplemented with computer simulations. The results led to the conclusion that attractive interactions between the nucleosomes existed which led to array compaction. In recent work [15], the interaction between nucleosomes was studied with the use of FRET. With this technique, transiently formed dinucleosomes with the lifetime $\sim 1 \mathrm{~s}$ were detected. The acetylation of histones and the presence of divalent cations decreased the stability of dinucleosomes.

Here, we apply AFM imaging to directly characterize the formation of tight dinucleosome complexes with the dinucleosome design. We showed that such complexes are formed but with low efficiency. The data are in line with the dynamic nature of dinucleosomes, in which the distance between the nucleosomes varies in a broad range, enabling the formation of close nucleosome-nucleosome contacts. We demonstrate the importance of environmental conditions to these dynamics and characterize the role of the ionic detergent CHAPS.

\section{Materials and Methods}

2.1. Preparation of Nucleosomal DNA. DNA for nucleosome assembly was generated by PCR using the plasmid pGEM3Z-601 as a template, which codes for a high-affinity nucleosome positioning sequence [16]. The PCR reaction (33 cycles of $94^{\circ} \mathrm{C} / 30 \mathrm{~s}, 54^{\circ} \mathrm{C} / 30 \mathrm{~s}, 72^{\circ} \mathrm{C} / 30 \mathrm{~s}$ ) was run in buffer containing $2.5 \mathrm{mM} \mathrm{MgCl}, 0.15 \mathrm{mM}$ dNTPs, and $0.016 \mathrm{U} / \mu \mathrm{L}$ of Taq DNA polymerase. Two PCR reactions were run with forward and reverse primers, respectively, set 1, primers CGGCCAGTGAATTGTAATACG and CGGTACGCTGGGTATGTGATGGACCCTATACG, and set 2, primers GCCATCCCCAGCCGGCAAGGTCGCTGTTCAAT and CGGGATCCTAATGACCAAGG. These reactions created products, which incorporated nonpalindromic BseYI restriction site at the ends. After restriction digest with BseYI and ligation with T4 DNA ligase, a DNA template of $560 \mathrm{bp}$ was obtained (see Figure S1 in Supplementary Material available online at doi:10.1155/2012/650840), which contained two nucleosome positioning sequences with a $60 \mathrm{bp}$ linker between them and arms of 127 and $79 \mathrm{bp}$ in length.

2.2. Histone Octamer Assembly and Purification. Histone octamers were assembled as follows [17]. Octamers were separated from tetramer and dimer fractions with size-exclusion chromatography (SEC) with Superdex 200 PC 3.2/30 column (GE Healthcare) at $4^{\circ} \mathrm{C}$. SEC fractions were analyzed for purity and histone stoichiometry using SDS-PAGE. The gel was stained using Coomassie Blue stain. Fractions containing histones $\mathrm{H} 2 \mathrm{~A}, \mathrm{H} 2 \mathrm{~B}, \mathrm{H} 3$, and $\mathrm{H} 4$ in approximately equal ratios were pooled and concentrated by centrifugation at $10,000 \mathrm{~g}$.

2.3. Nucleosome Refolding. Dinucleosome particle samples (di-NCP) were prepared as described earlier $[18,19]$. Briefly, histone octamers and DNA containing the nucleosome positioning sequence were mixed in equimolar concentrations in $2 \mathrm{M} \mathrm{NaCl}$ and kept for $30 \mathrm{~min}$ at RT. A dilution series was prepared using $10 \mathrm{mM}$ Tris $\mathrm{HCl}$ to produce final concentrations of $1 \mathrm{M}, 0.67 \mathrm{M}$, and $0.5 \mathrm{M} \mathrm{NaCl}$. Diluted samples were kept at $4{ }^{\circ} \mathrm{C}$ for $1 \mathrm{~h}$ before dialysis against one change of volume of $0.2 \mathrm{M} \mathrm{NaCl}$ overnight. Nucleosomes were concentrated using Microcon centrifugal filter devices, MWCO 10,000 at 7,000 g for $10 \mathrm{~min}$ at $4^{\circ} \mathrm{C}$ and dialyzed against one change of $200 \mathrm{~mL}$ of buffer containing $10 \mathrm{mM}$ Hepes- $\mathrm{NaCl}$, $\mathrm{pH} 7.5$, and $1 \mathrm{mM}$ EDTA for $3 \mathrm{~h}$ at $4^{\circ} \mathrm{C}$.

2.4. Atomic Force Microscopy. Freshly cleaved mica was modified with a $167 \mu \mathrm{M}$ solution of 1-(3-aminopropyl)-silatrane (APS) for $30 \mathrm{~min}$ at room T to make APS-mica as described previously in [19-21]. The nucleosome stock solution was diluted into $10 \mathrm{mM}$ Tris- $\mathrm{HCl}, \mathrm{pH} 7.5,4 \mathrm{mM}, \mathrm{MgCl}_{2}$ buffer, and $5 \mu \mathrm{L}$ of the solution was deposited on APS-treated mica for 3 minutes, washed with deionized water, and dried under argon flow. AFM images were collected on an MM AFM/NanoScope IIId system (Bruker-Nano/Veeco, Santa Barbara, Calif, USA) as described in [19, 20, 22].

2.5. Measurement of Nucleosome Parameters. The samples deposited on APS mica were analyzed with FemtoScan software. The following 5 initial parameters were measured: length of each DNA arm, angle between arms (interarm angle), height of the nucleosome core particle, and diameter as the width of the nucleosome core particle at half height. The length of DNA was measured with FemtoScan software using the "curve" parameter. The length of wrapped DNA was measured by subtracting the sum of both DNA arms and the linker from the length of free DNA measured on the same sample (see Figure 1). The length of the linker DNA was measured from the center of one nucleosome along the linker to the center of another nucleosome minus $5 \mathrm{~nm}$ (the size of the nucleosome particle itself).

\section{Results}

3.1. Visualization of Dinucleosomes with AFM. The diNCP sample was imaged similarly to the mononucleosome sample described earlier [18-22]. Diluted samples $(0.8 \mathrm{nM}$ concentration in $10 \mathrm{mM}$ Tris- $\mathrm{HCl}, \mathrm{Ph} 7.5$, and $4 \mathrm{mM} \mathrm{MgCl}_{2}$ buffer) were deposited on APS mica and after drying, imaged in air at ambient conditions. Figure 1 shows a typical AFM image of the dinucleosome sample. The nucleosomes appear as two bright globular features (blobs) separated with a DNA region (linker) with two free DNA arms at each nucleosome. The yield of dinucleosomes in the sample is high. The image in Figure 1 has 6 dinucleosomes and only one free DNA. 


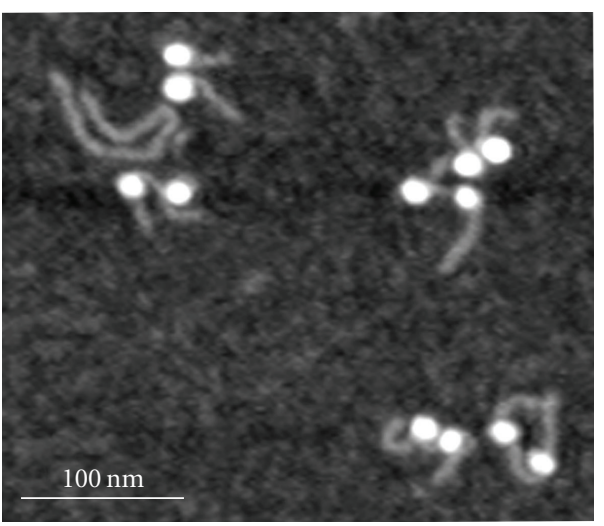

Figure 1: AFM images of dinucleosomes obtained with the use of APS mica. AFM images were acquired with the use of the MM AFM/NanoScope IV system operating in Tapping Mode.

3.2. Nucleosome Wrapping in the Dinucleosomes. Images as shown in Figure 1 were used for assessing the degree of nucleosome wrapping. This value was calculated from the measurements of the lengths of the DNA arms and the linker DNA as described in Section 2. The histogram for this value is shown in Figure 2. The distribution is rather broad with a maximum for the Gaussian $=107 \mathrm{~nm}$. The expected length of wrapped DNA for the di-NCP is $147(\mathrm{bp}) \times 0.32(\mathrm{~nm} / \mathrm{bp}) \times$ $2=94.08 \mathrm{~nm}$. Due to the tip convolution effect, NCPs on the AFM images appear $\sim 10 \mathrm{~nm}$ larger [19]. This effect leads to a decrease of the length of the measured unwrapped DNA by $\sim 10 \mathrm{~nm}$; therefore, given the convolution effect, the measured mean value of wrapped DNA is close to the expected value. At the same time, the distribution of the lengths is broad, ranging between $\sim 86 \mathrm{~nm}$ and $127 \mathrm{~nm}$ (mean values). This suggests that nucleosomes are dynamic, enabling the formation of particles with varied amounts of DNA wrapping, which is in line with our previous study of mononucleosomes $[18,19,22]$.

\subsection{The Internucleosome Distance and the Interaction between} Nucleosome Cores. To address the question of whether nucleosome core particles interact with each other in the absence of $\mathrm{H} 1$ histone, we measured two parameters of dinucleosomes, the length of the linker, and the shortest internucleosome distance. The first parameter will answer the question of whether the interaction occurs via sliding of nucleosomes. The second parameter will reveal whether the nucleosomes interact in space without sliding.

A subset of three images of dinucleosomes with different relative positions is shown in Figure 3. Frame "a" on this set corresponds to dinucleosomes with very small internucleosomal distances. The distance is considerably larger for the dinucleosome in Figure 3(b) $(\sim 17 \mathrm{~nm})$ and the last image (Figure 3(c)) corresponds to a linker length $\sim 37 \mathrm{~nm}$.

The linker length measurements are shown in Figure 4(a). In addition to the main peak at the maximum $\sim 19 \mathrm{~nm}$, there is a minor peak at $\sim 35 \mathrm{~nm}$. The appearance of the second peak suggests that there is a population of partially unwrapped nucleosomes. This interpretation is in line with

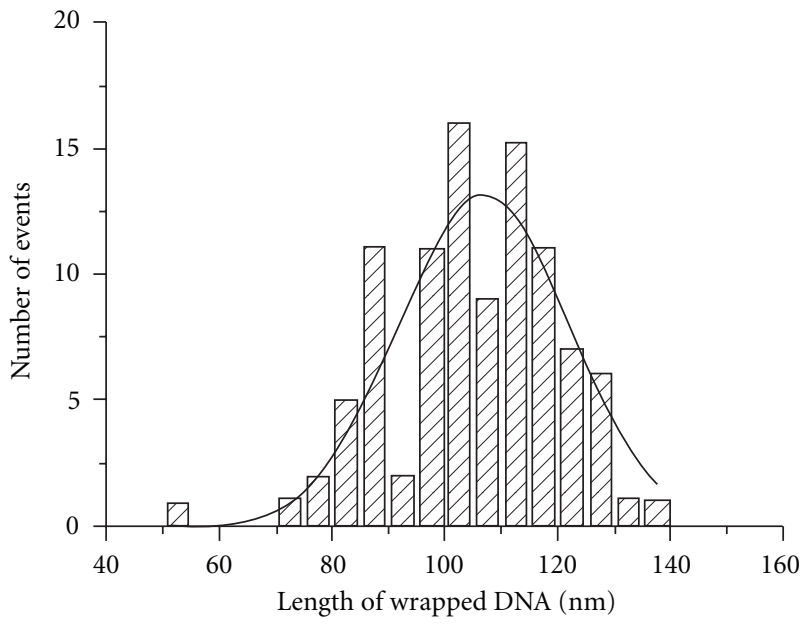

FIgURE 2: The length distribution for wrapped DNA for all dinucleosome molecules in the population. The value of total DNA wrapped around both octamers was calculated by subtracting the measured length of two arms and a linker from the total DNA length, as described in Section 2. The maximum of the Gaussian corresponds to $106.6 \pm 2 \mathrm{~nm}$.

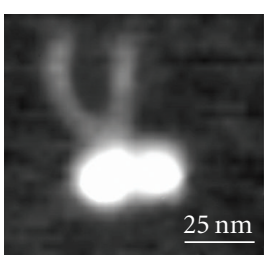

(a)

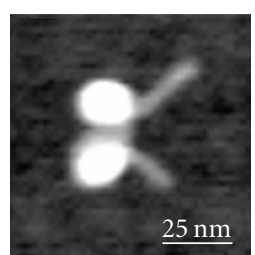

(b)

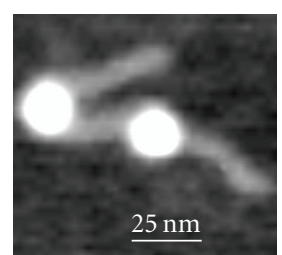

(c)
FIGURE 3: Selected images of dinucleosomes with different internucleosomal distances.

measurements of wrapped DNA. The most important finding of this analysis is that the measured linker length $(\sim 19 \mathrm{~nm})$ is very close to the expected value, $19.84 \mathrm{~nm}$.

The shortest distance between the nucleosomes was obtained by measuring the center-to-center distance. The results are assembled in Figure 4(b). The dinucleosomes with visually low wrapping were excluded from this analysis. The histogram has a maximum at $\sim 22 \mathrm{~nm}$ and a low percentage of nucleosomes with the internucleosomal distance of $10 \mathrm{~nm}$. Thus, both measurements do not support the idea of interactions between the nucleosomes.

3.4. Effect of the Zwitterionic Detergent, CHAPS, on the Morphology of Dinucleosomes. Nucleosomes are unstable at low concentrations (nanomolar range), and detergents are used to stabilize them (e.g., [23]). We have shown recently that the zwitterionic detergent, CHAPS, considerably increases the stability of mononucleosomes at the nanomolar concentration and works in a broad range of ionic strengths [18]. Importantly, the sequence-specific positioning of the nucleosomes also changes in the presence of CHAPS. We analyzed the effect of CHAPS for the dinucleosome samples 


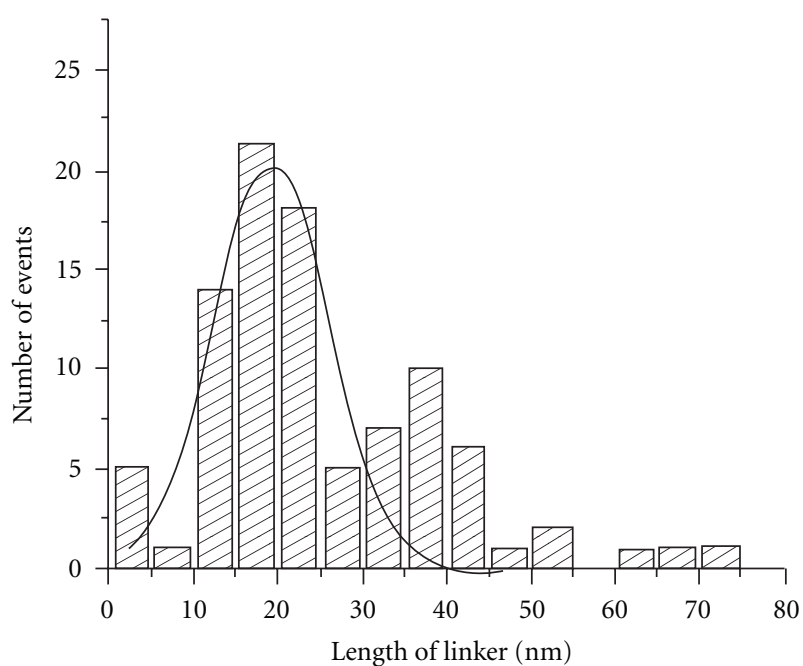

(a)

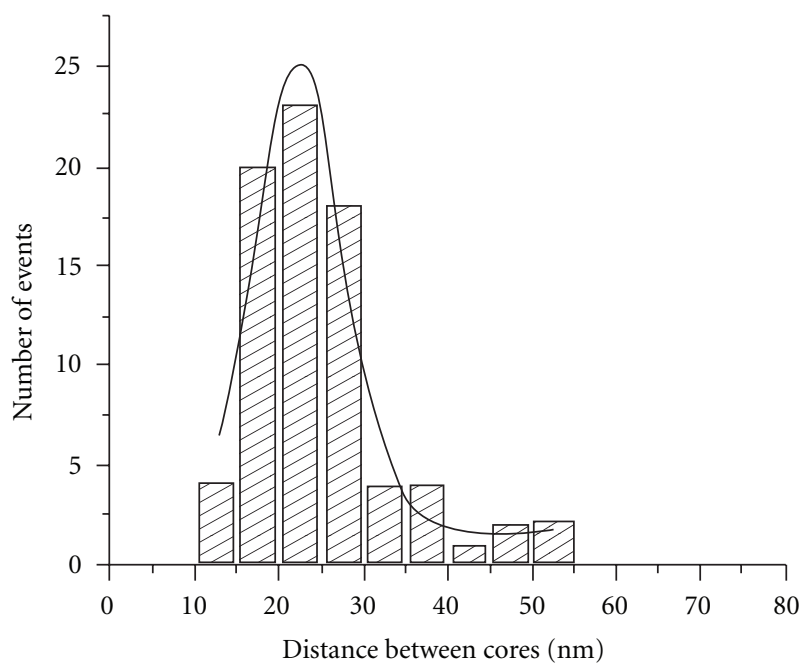

(b)

FIgURE 4: Distributions of the linker lengths (a) and the internucleosomal distances (b). Maximum of the Gaussian for the linker length is $18.9 \mathrm{~nm}$. Maximum of the Gaussian for distances between cores is $22.2 \mathrm{~nm}$.

to understand whether CHAPS changes the stability and morphology of dinucleosomes.

Images in Figure 5 are the typical images obtained for the dinucleosome sample incubated in Tris- $\mathrm{HCl}$ buffer $(10 \mathrm{mM}$ Tris- $\mathrm{HCl}, \mathrm{pH} 7.5,4 \mathrm{mM} \mathrm{MgCl}_{2}, \mathrm{pH} 7.5$ ) at the concentration $0.8 \mathrm{nM}$ for $30 \mathrm{~min}$ in the absence of CHAPS (a) and in the presence of $1.6 \mathrm{mM}$ CHAPS (b). The initial sample contained $85 \%$ of dinucleosomes and appeared under AFM as the sample shown in Figure 1. Incubation for $30 \mathrm{~min}$ (Figure 5(a)) leads to almost complete dissociation of the nucleosomes from the DNA template; therefore, only naked DNA is seen under AFM. In contrast, dinucleosomes remain intact if $1.6 \mathrm{mM}$ CHAPS is present (Figure 5(b)). A similar analysis was performed for other incubation times, and the data are assembled in Figure S2. This graph shows that in the absence of CHAPS, nucleosomes dissociate rapidly with a characteristic time $\sim 7.5 \mathrm{~min}$, whereas in the presence of CHAPS, dinucleosomes are stable after incubation for 48 hours. Similar data were obtained for the mononucleosomes [18], suggesting that each nucleosome in the dinucleosome design behaves independently.

We also measured the variation of the linker length over incubation time with $1.6 \mathrm{mM}$ CHAPS. Initially at zero incubation time, both samples were indistinguishable with a linker length $\sim 20 \mathrm{~nm}$. Moreover, the distributions of the linker lengths for the control sample and the sample prepared in the presence of CHAPS were very close (Figure S3). The time-dependent measurements of the linker lengths for the CHAPS containing samples showed dramatic changes in the width distributions over time. The values change in the order of $11.4 \mathrm{~nm}, 22.3 \mathrm{~nm}, 23.1 \mathrm{~nm}$, and $18.1 \mathrm{~nm}$ for times $0 \mathrm{~min}, 5 \mathrm{~min}, 2$ hours, and 48 hours, respectively. The histograms for each incubation time are shown in Figure S4. These findings suggest that the sequence specific formation of nucleosomes, in the presence of CHAPS, is less stringent.

\section{Discussion}

The data obtained in this paper show that the direct interaction of two nucleosomes separated by a linker in the absence of $\mathrm{H} 1$ histone can occur, but the probability of such events is rather low. We were able to identify by AFM complexes with a small internucleosomal distance similar to the one shown in Figure 3(a). However, the yield of such complexes according to the statistical analysis is low (Figure 4(b)). At the same time, the AFM analysis shows that nucleosomes with a broad range of internucleosomal distances are formed, suggesting that nucleosomal particles in the di-nucleosomal construct are rather dynamic, enabling the formation of systems with different distances between the nucleosomes. The highly dynamic feature of nucleosomes was directly proven in our early work with mononucleosomes, in which the dynamics were observed directly with time-lapse AFM [19]. The findings in this paper are in line with our previous studies. Therefore, nucleosomes can translocate along the DNA, approach quite close to each other or move apart. The interaction between two nucleosomes was observed in [15], but these were free nucleosomes, and, in the complexes, the two nucleosomes were separated by approximately $2 \mathrm{~nm}$. In addition, according to [15], the dinucleosome complexes are formed transiently, which is in line with our model of nucleosome dynamics.

There are two models for the formation of tight dinucleosome complexes, translocation of nucleosomes, and their interaction in space via bending of the linker. The second mechanism requires substantial deformation of the linker. To make a close contact, a U-turn deformation is needed, which is problematic for a linker as short as $60 \mathrm{bp}$. Deformations of this kind require a segment with a random sequence 4 times larger in length [24]. Internucleosomal interactions were observed in [14], in which arrays of nucleosomes were used enabling the interaction of nucleosomes distantly located in the array. Therefore, we assume that translocation of 


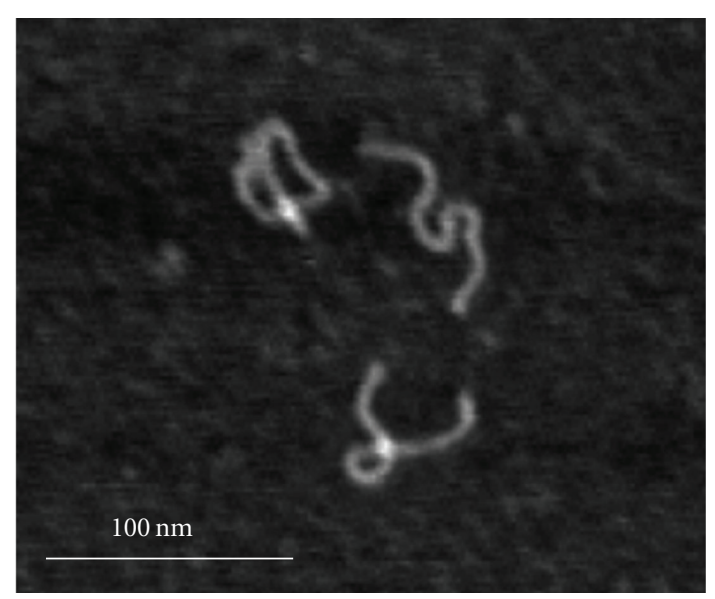

(a)

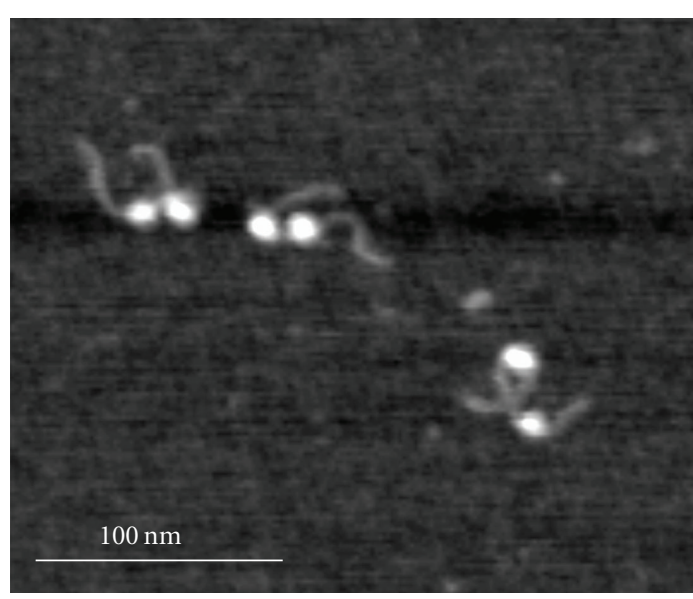

(b)

FIgURE 5: AFM images of the dinucleosome sample incubated in Tris- $\mathrm{HCl}$ buffer (10 mM Tris- $\left.\mathrm{HCl}, \mathrm{pH} 7.5,4 \mathrm{mM} \mathrm{MgCl}_{2}\right)$. The nucleosomes concentration was $0.8 \mathrm{nM}$, the sample was incubated at $4^{\circ} \mathrm{C}$ for $30 \mathrm{~min}$ without CHAPS (a) and with CHAPS (b).

the nucleosome is the mechanism by which close contacts between the nucleosomes are made.

There are two models for nucleosome dynamics, sliding and site transfer requiring unwrapping of both arms of the nucleosome $[25,26]$. Our previous findings [19] were in favor of the site transfer model. Indeed, the dynamics via unwrapping of both arms of the nucleosome was the only pathway imaged with time-lapse AFM directly. However, recently we applied high-speed AFM to directly image nucleosome dynamics [27]. These studies enabled us to observe transient sliding of the nucleosome at the subsecond time scale. The nucleosome moved from the original sitespecific position and returned back to the same position. Note that this and our previous studies were performed with the use of the 601 sequence, characterized by a high affinity for nucleosome formation. Therefore, we assume that transient formation of tight di-nucleosomal complexes occurs via a sliding mechanism.

According to the statistical analysis (Figure 4(a)), the lengths of the linker for different nucleosomes vary over $15 \mathrm{~nm}$. However, the range is two times wider if CHAPS is added (Figure S4), suggesting that the dynamics of nucleosomes is much broader if CHAPS is present. We have shown recently (see [27]) that CHAPS facilitates nucleosome dynamics and sliding away from the 601 motif into regions with a random sequence occurs with the formation of stable nonspecific nucleosomal particles [18]. We hypothesized that in the presence of CHAPS, the stringency of the sequence specificity for nucleosome formation is lowered, enabling the formation of stably existing nucleosomes over the region away from the 601 motif [18]. The broad variability of the internucleosomal linker is in line with this hypothesis.

Although CHAPS is not a natural product, it has similarity in structure with cholesterol. Cholesterol has the same conjugated 3 benzyl rings and 1 pentyl ring as CHAPS. Also, the content of cholesterol in nuclear membranes is about $10 \%$. This suggests that the possible involvement of cholesterol, as a significant component of the nuclear membrane, in the regulation of chromatin dynamics should be taken into account. Importantly, a modified water-soluble cholesterol, cholesteryl sulfate, is a minor constituent of various cell types including erythrocytes [28] and spermatozoa [29]. It was shown that cholesteryl sulfate is also present in blood plasma [30]. We have shown [18] that cholesteryl sulfate also stabilizes nucleosomes and its participation in chromatin dynamics needs to be considered as well.

\section{Acknowledgments}

The authors thank L. Shlyakhtenko for valuable discussions and insightful suggestions and J. Widom and G. Narlikar for useful comments on the results. The work is supported by grants from the DOE (DE-FG02-08ER64579), NATO (SfP 983204), and the Nebraska Research Initiative (NRI, all to YLL).

\section{References}

[1] J. C. Hansen, "Conformational dynamics of the chromatin fiber in solution: determinants, mechanisms, and functions," Annual Review of Biophysics and Biomolecular Structure, vol. 31, pp. 361-392, 2002.

[2] F. Thoma, Koller Th., and A. Klug, "Involvement of histone $\mathrm{H} 1$ in the organization of the nucleosome and of the saltdependent superstructures of chromatin," Journal of Cell Biology, vol. 83, no. 2, pp. 403-427, 1979.

[3] M. Garcia-Ramirez, F. Dong, and J. Ausio, "Role of the histone 'tails' in the folding of oligonucleosomes depleted of histone H1," The Journal of Biological Chemistry, vol. 267, no. 27, pp. 19587-19595, 1992.

[4] C. L. Woodcock and S. Dimitrov, "Higher-order structure of chromatin and chromosomes," Current Opinion in Genetics and Development, vol. 11, no. 2, pp. 130-135, 2001.

[5] K. Luger, A. W. Mäder, R. K. Richmond, D. F. Sargent, and T. J. Richmond, "Crystal structure of the nucleosome core particle at $2.8 \AA$ resolution,” Nature, vol. 389 , no. 6648, pp. 251-260, 1997. 
[6] B. Dorigo, T. Schalch, K. Bystricky, and T. J. Richmond, "Chromatin fiber folding: requirement for the histone $\mathrm{H} 4 \mathrm{~N}$ terminal tail," Journal of Molecular Biology, vol. 327, no. 1, pp. 85-96, 2003.

[7] B. Dorigo, T. Schalch, A. Kulangara, S. Duda, R. R. Schroeder, and T. J. Richmond, "Nucleosome arrays reveal the two-start organization of the chromatin fiber," Science, vol. 306, no. 5701, pp. 1571-1573, 2004.

[8] J. Y. Fan, D. Rangasamy, K. Luger, and D. J. Tremethick, "H2A.Z alters the nucleosome surface to promote HP1 $\alpha$ mediated chromatin fiber folding," Molecular Cell, vol. 16, no. 4, pp. 655-661, 2004.

[9] C. Tse and J. C. Hansen, "Hybrid trypsinized nucleosomal arrays: identification of multiple functional roles of the $\mathrm{H} 2 \mathrm{~A} / \mathrm{H} 2 \mathrm{~B}$ and $\mathrm{H} 3 / \mathrm{H} 4 \mathrm{~N}$-termini in chromatin fiber compaction," Biochemistry, vol. 36, no. 38, pp. 11381-11388, 1997.

[10] L. M. Carruthers and J. C. Hansen, "The core histone N termini function independently of linker histones during chromatin condensation," The Journal of Biological Chemistry, vol. 275, no. 47, pp. 37285-37290, 2000.

[11] F. Gordon, K. Luger, and J. C. Hansen, "The core histone $\mathrm{N}$-terminal tail domains function independently and additively during salt-dependent oligomerization of nucleosomal arrays," The Journal of Biological Chemistry, vol. 280, no. 40, pp. 33701-33706, 2005.

[12] G. Arya and T. Schlick, "A tale of tails: how histone tails mediate chromatin compaction in different salt and linker histone environments," Journal of Physical Chemistry A, vol. 113, no. 16, pp. 4045-4059, 2009.

[13] P. Y. Kan, T. L. Caterino, and J. J. Hayes, "The H4 tail domain participates in intra- And internucleosome interactions with protein and DNA during folding and oligomerization of nucleosome arrays," Molecular and Cellular Biology, vol. 29, no. 2, pp. 538-546, 2009.

[14] F. Montel, H. Menoni, M. Castelnovo et al., "The dynamics of individual nucleosomes controls the chromatin condensation pathway: direct atomic force microscopy visualization of variant chromatin," Biophysical Journal, vol. 97, no. 2, pp. 544$553,2009$.

[15] J. Y. Lee, S. Wei, and T. -H. Lee, "Effects of histone acetylation by Piccolo NuA4 on the structure of a nucleosome and the interactions between two nucleosomes," The Journal of Biological Chemistry, vol. 286, no. 13, pp. 11099-11109, 2011.

[16] P. T. Lowary and J. Widom, "New DNA sequence rules for high affinity binding to histone octamer and sequence-directed nucleosome positioning," Journal of Molecular Biology, vol. 276, no. 1, pp. 19-42, 1998.

[17] P. N. Dyer, R. S. Edayathumangalam, C. L. White et al., "Reconstitution of nucleosome core particles from recombinant histones and DNA," Methods in Enzymology, vol. 375, pp. 23-44, 2004.

[18] I. Menshikova, E. Menshikov, N. Filenko, and Y. L. Lyubchenko, "Nucleosomes structure and dynamics: effect of CHAPS," International Journal of Biochemistry and Molecular Biology, vol. 2, no. 2, pp. 129-137, 2011.

[19] L. S. Shlyakhtenko, A. Y. Lushnikov, and Y. L. Lyubchenko, "Dynamics of nucleosomes revealed by time-lapse atomic force microscopy," Biochemistry, vol. 48, no. 33, pp. 78427848, 2009.

[20] Y. L. Lyubchenko and L. S. Shlyakhtenko, "AFM for analysis of structure and dynamics of DNA and protein-DNA complexes," Methods, vol. 47, no. 3, pp. 206-213, 2009.
[21] L. S. Shlyakhtenko, A. A. Gall, A. Filonov, Z. Cerovac, A. Lushnikov, and Y. L. Lyubchenko, "Silatrane-based surface chemistry for immobilization of DNA, protein-DNA complexes and other biological materials," Ultramicroscopy, vol. 97, no. 1-4, pp. 279-287, 2003.

[22] N. A. Filenko, C. Kolar, J. T. West et al., "The role of histone $\mathrm{H} 4$ biotinylation in the structure of nucleosomes," PLoS One, vol. 6, no. 1, article e16299, 2011.

[23] W. J. A. Koopmans, A. Brehm, C. Logie, T. Schmidt, and J. Van Noort, "Single-pair FRET microscopy reveals mononucleosome dynamics," Journal of Fluorescence, vol. 17, no. 6, pp. 785-795, 2007.

[24] S. Geggier and A. Vologodskii, "Sequence dependence of DNA bending rigidity," Proceedings of the National Academy of Sciences of the United States of America, vol. 107, no. 35, pp. 15421-15426, 2010.

[25] G. Li, M. Levitus, C. Bustamante, and J. Widom, "Rapid spontaneous accessibility of nucleosomal DNA," Nature Structural and Molecular Biology, vol. 12, no. 1, pp. 46-53, 2005.

[26] G. Li and J. Widom, "Nucleosomes facilitate their own invasion," Nature Structural and Molecular Biology, vol. 11, no. 8, pp. 763-769, 2004.

[27] A. Miyagi, T. Ando, and Y. L. Lyubchenko, "Dynamics of nucleosomes assessed with time-lapse high-speed atomic force microscopy," Biochemistry, vol. 50, no. 37, pp. 7901-7908, 2011.

[28] F. A.J. Muskiet, G. Jansen, and B. G. Wolthers, "Gas-chromatographic determination of cholesterol sulfate in plasma and erythrocytes, for the diagnosis of recessive X-linked ichthyosis," Clinical Chemistry, vol. 29, no. 7, pp. 1404-1407, 1983.

[29] J. Langlais, M. Zollinger, and L. Plante, "Localization of cholesteryl sulfate in human spermatozoa in support of a hypothesis for the mechanism of capacitation," Proceedings of the National Academy of Sciences of the United States of America, vol. 78, no. 12, pp. 7266-7270, 1981.

[30] E. Gurpide, K. D. Roberts, M. T. Welch, L. Bandy, and S. Lieberman, "Studies on the metabolism of blood-borne cholesterol sulfate,” Biochemistry, vol. 5, no. 10, pp. 3352-3362, 1966. 

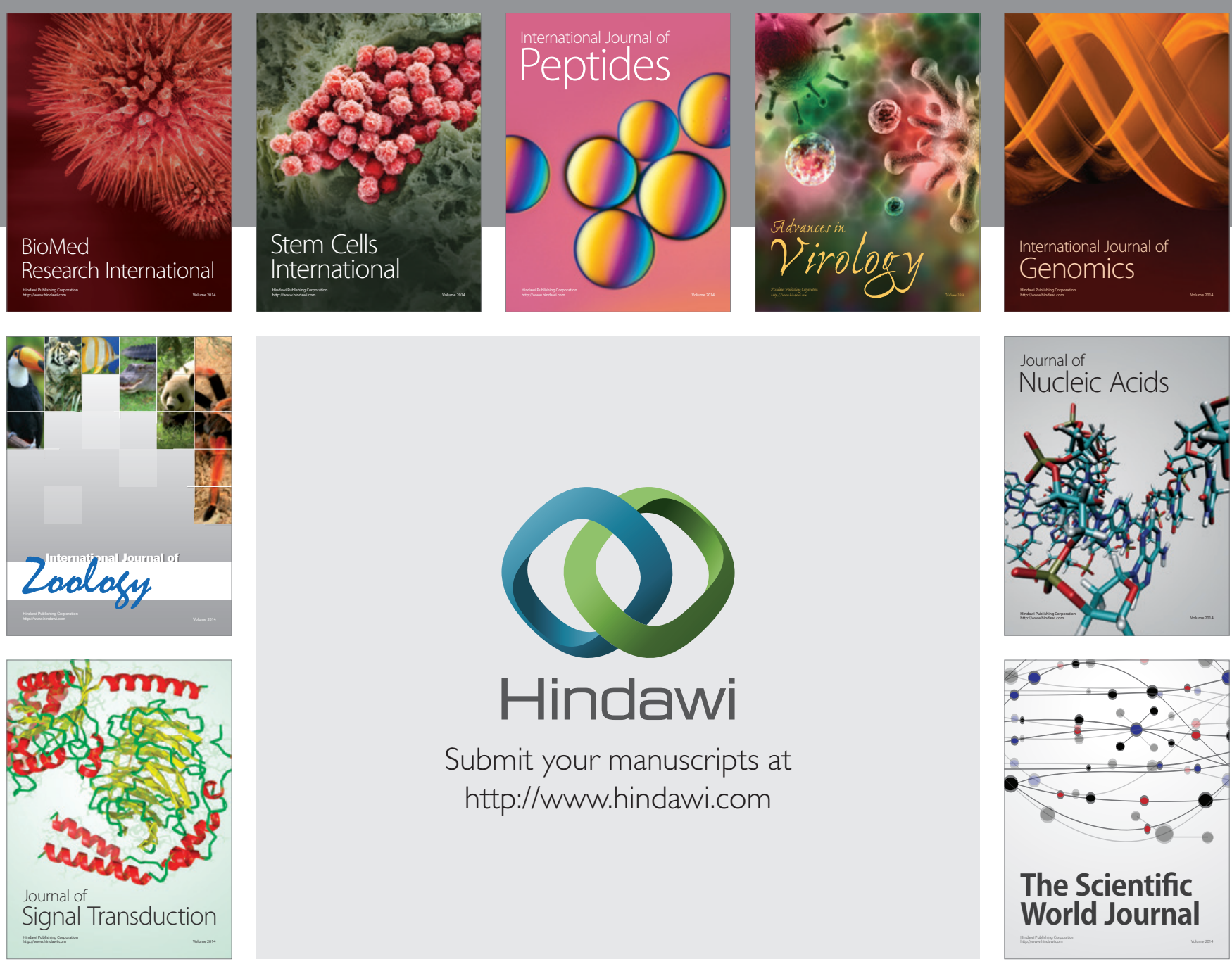

Submit your manuscripts at

http://www.hindawi.com
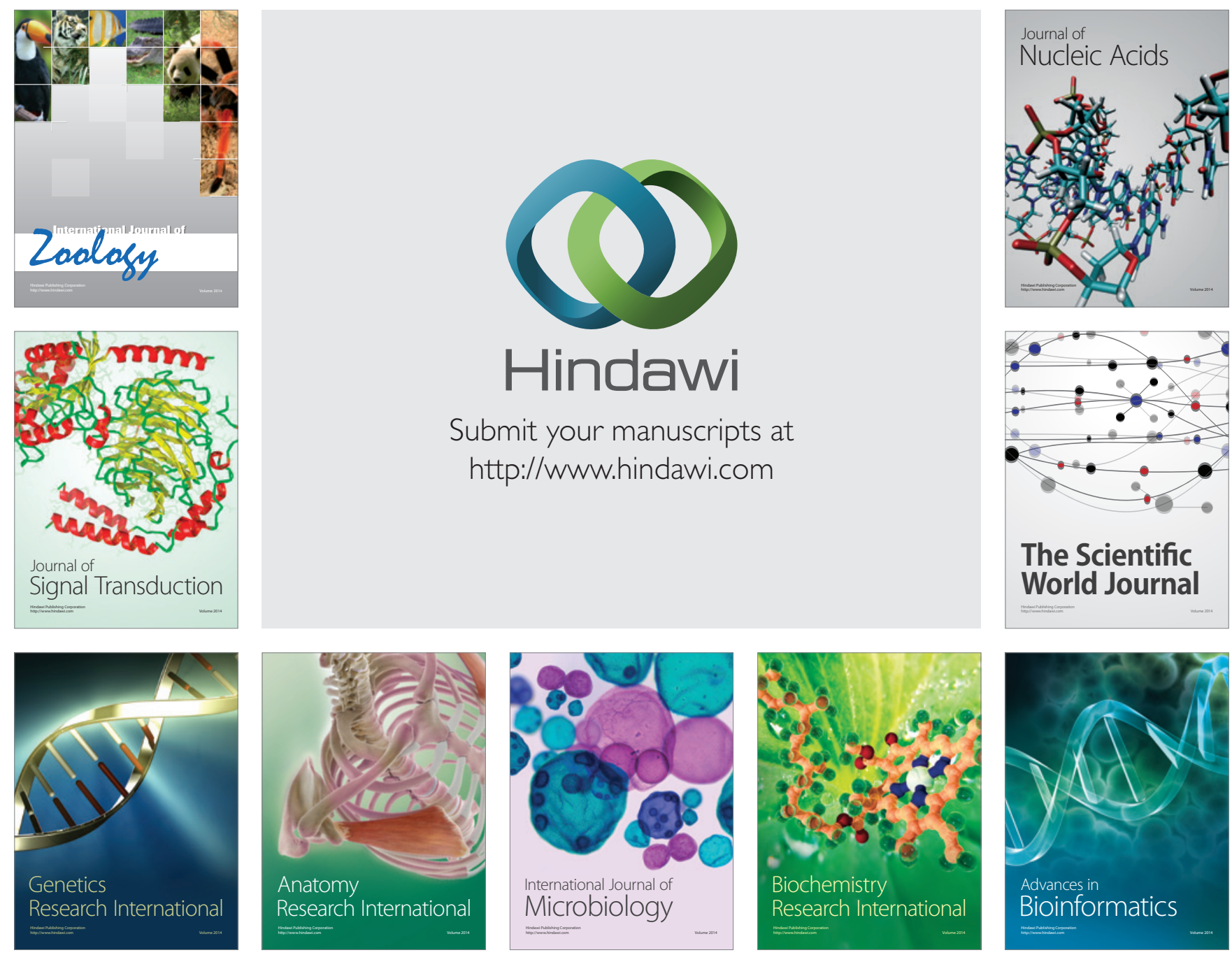

The Scientific World Journal
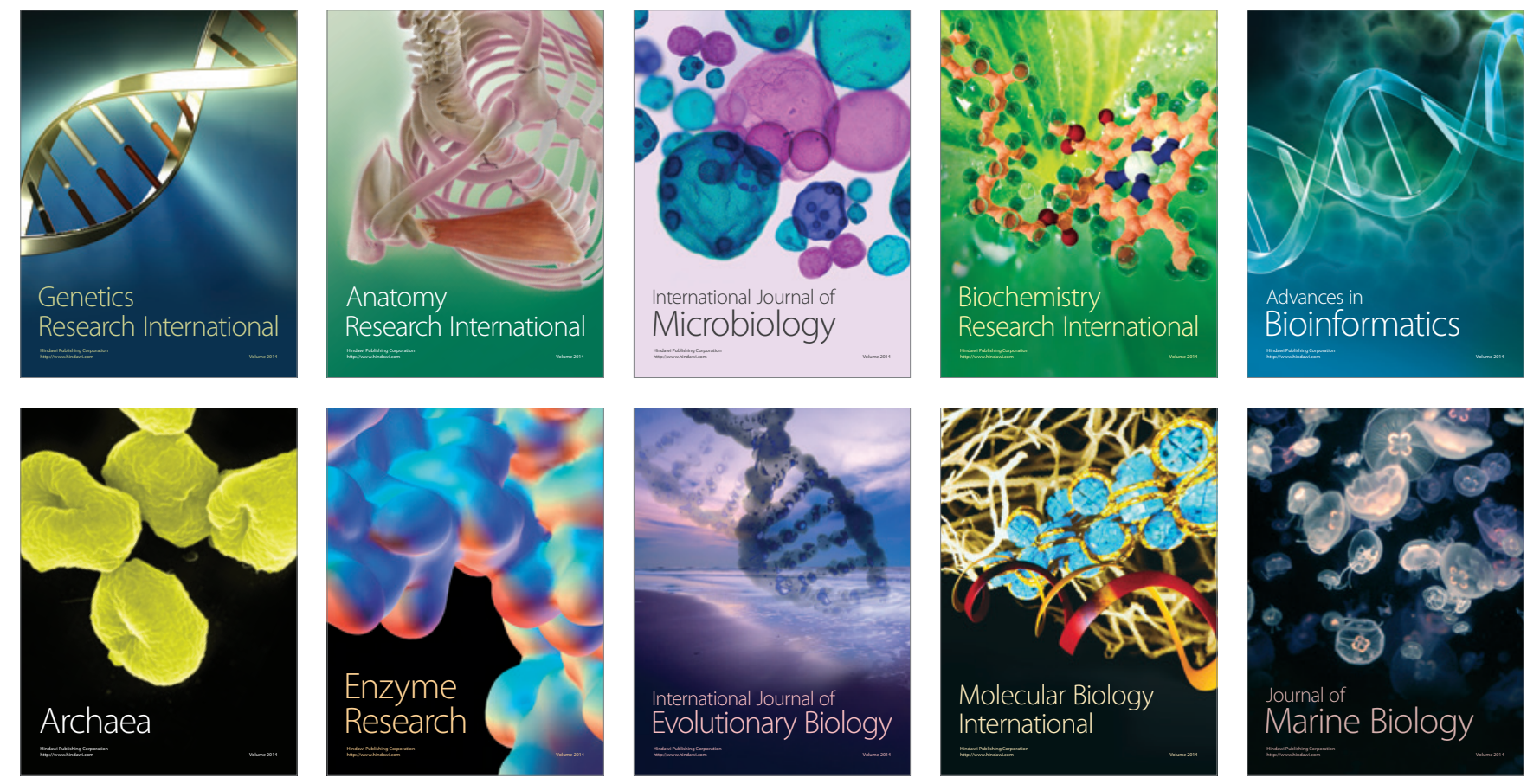